\title{
Malnutrition among older adults living in Portuguese nursing homes: the PEN-3S study
}

Teresa Madeira ${ }^{1,2,3, *}$, Catarina Peixoto-Plácido 1,2,3, Nuno Sousa-Santos 1,2,3 Osvaldo Santos 1,2,3, Violeta Alarcão 1,2,3, Beatriz Goulão ${ }^{4}$, Nuno Mendonça '1,5, Paulo Jorge Nicola 1,2,3, Agneta Yngve ${ }^{6}$, Asta Bye ${ }^{7,8}$, Astrid Bergland7 , Teresa F Amaral ${ }^{9}$, Carla Lopes $^{10,11}$ and João Gorjão Clara ${ }^{1,2,3}$

${ }^{1}$ Instituto de Medicina Preventiva e Saúde Pública, Faculdade de Medicina, Universidade de Lisboa, Avenida Professor Egas Moniz, 1649-028 Lisboa, Portugal: ${ }^{2}$ Instituto de Saúde Ambiental, Faculdade de Medicina, Universidade de Lisboa, Lisboa, Portugal: ${ }^{3}$ Faculdade de Medicina, Universidade de Lisboa, Lisboa, Portugal: ${ }^{4}$ Health Services Research Unit, University of Aberdeen, Aberdeen, UK: ${ }^{5}$ Institute for Ageing, Newcastle University, Newcastle upon Tyne, UK: ${ }^{6}$ Department of Food, Nutrition and Dietetics, Uppsala University, Uppsala, Sweden: ${ }^{7}$ OsloMet - Oslo Metropolitan University, Oslo, Norway: ${ }^{8}$ Regional Advisory Unit for Palliative Care, Department of Oncology, Oslo University Hospital, Oslo, Norway: ${ }^{9}$ Faculdade de Ciências da Nutrição e Alimentação, Universidade do Porto, Porto, Portugal: ${ }^{10}$ EPIUnit - Institute of Public Health, University of Porto, Porto, Portugal:

${ }^{11}$ Department of Public Health and Forensic Sciences and Medical Education, Faculty of Medicine, University of Porto, Porto, Portugal

Submitted 20 October 2017: Final revision received 30 June 2018: Accepted 7 August 2018: First published online 15 0ctober 2018

\begin{abstract}
Objective: To characterise the nutritional status and to identify malnutritionassociated variables of older adults living in Portuguese nursing homes.

Design: Cross-sectional study. Data on demographic and socio-economic characteristics, self-reported morbidity, eating-related problems, nutritional status, cognitive function, depression symptoms, loneliness feelings and functional status were collected by trained nutritionists through a computer-assisted face-to-face structured interview followed by standardised anthropometric measurements. Logistic regression was used to identify factors associated with being at risk of malnutrition/malnourished.

Setting: Portuguese nursing homes.

Subjects: Nationally representative sample of the Portuguese population aged 65 years or over living in nursing homes.

Results: A total of 1186 individuals (mean age 83.4 years; $72 \cdot 8 \%$ women) accepted to participate. According to the Mini Nutritional Assessment, 4.8 (95\% CI 3.2, 7.3) \% were identified as malnourished and 38.7 (95\% CI 33.5, 44.2) \% were at risk of malnutrition. These percentages increased with age and were significantly higher for women. Logistic regression showed (OR; 95\% CI) that older adults reporting no or little appetite $(6 \cdot 5 ; 2 \cdot 7,15 \cdot 3)$, those revealing symptoms of depression $(2 \cdot 6 ; 1 \cdot 6,4 \cdot 2)$ and those who were more dependent in their daily living activities $(4 \cdot 7 ; 2 \cdot 0,11 \cdot 1)$ were also at higher odds of being malnourished or at risk of malnutrition.

Conclusions: Malnutrition and risk of malnutrition are prevalent among nursing home residents in Portugal. It is crucial to routinely screen for nutritional disorders, as well as risk factors such as symptoms of depression and lower functional status, to prevent and treat malnutrition.
\end{abstract}

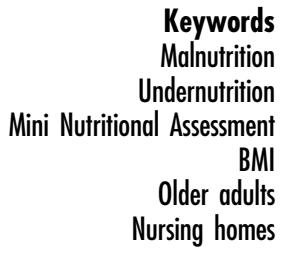

Malnutrition Undernutrition

Older adults Nursing homes
Nutrition is a key health determinant that impacts intrinsic capacity and quality of life among older adults ${ }^{(1-3)}$. During the ageing process, food intake and consequently nutritional status may be compromised by changes in sensory functions, mastication and deglutition capacities, and gastrointestinal physiology, as well as by progressive loss of vision and hearing ${ }^{(4-6)}$. In addition, chronic conditions (e.g. cancer and dementia), polypharmacy ${ }^{(7-9)}$, psychosocial (e.g. depression and self-perceived loneliness) and environmental factors (e.g. financial difficulties and living 
alone $)^{(10-12)}$, independently or combined, may greatly influence dietary patterns as people age. These aspects, combined with specific nutritional requirements and agerelated decline in appetite and consequently food intake, increase the risk of malnutrition among older adults ${ }^{(13)}$.

Malnutrition refers to deficiencies, excess or imbalance in intake of energy and/or nutrients. The present paper focuses on malnutrition due to starvation, disease or advanced ageing, which can be defined as 'a state resulting from lack of uptake or intake of nutrition leading to altered body composition (decreased body fat mass) and body cell mass leading to diminished physical and mental function and impaired clinical outcome from disease, ${ }^{,(14)}$. Diagnostic criteria for malnutrition are not consensual in the scientific community, which results in considerable methodological differences among epidemiological and clinical studies and prevents direct comparison between them. To fill this gap, the European Society of Clinical Nutrition and Metabolism recently recommended the use of BMI, or alternatively unintentional weight loss combined with either reduced BMI (with age-dependent cutoffs) or reduced fat-free mass, to define malnutrition. It was also stated that before diagnosis, it is mandatory that the individual meets the criteria for being at risk of malnutrition by a validated risk screening tool, such as the Mini Nutritional Assessment $\left(\text { MNA }^{\circledR}\right)^{(15)}$.

Malnutrition is associated with negative health outcomes, including increased risk of falls, immune dysfunction, decreased quality of life, more and longer hospitalisations, and ultimately higher mortality ${ }^{(16-19)}$. To worsen this scenario, malnutrition risk factors, such as depression and cognitive impairment, are also becoming more prevalent as the world's population ages. These conditions increase disability, thus impacting the individual's intrinsic capacity to continue to be and to do what $\mathrm{s} / \mathrm{he}$ values $^{(7,20,21)}$. Additionally, the association between malnutrition and disability can work both ways: malnourished individuals are more likely to be dependent in activities of daily living, and disabled individuals are at greater risk for nutritional problems given their greater dependence on others $^{(3,22)}$.

Despite the aforementioned lack of consensus on diagnostic criteria and the absence of a gold standard concerning screening tools, malnutrition has been identified as a prevalent problem in older adults, particularly among those living in nursing homes ${ }^{(23)}$. A literature review based on fifty-three studies ( $n$ 50 957) that used the same screening tool $\left(\mathrm{MNA}^{\circledR}\right)$ concluded that $4.2 \%$ of community-dwelling older adults were malnourished and $27.4 \%$ were at risk of malnutrition ${ }^{(24)}$. However, when considering nursing home residents (seventy-three studies, $n 20410$ ), the prevalence was even higher, reaching $27 \cdot 2$ and $52 \cdot 1 \%$, respectively ${ }^{(24)}$.

Differences in autonomy, cognitive function and emotional state between well-nourished and malnourished individuals seem to be more pronounced among those who live in nursing homes ${ }^{(12)}$. Structural factors (type of funding, management, capacity or location) of the nursing home may impact malnutrition prevalence. Interventions targeted at improving nutrition in nursing homes have been identified as a priority ${ }^{(25)}$. In fact, interventions aimed at reverting weight loss, including fat-free mass loss, have proved to be effective if administered in time ${ }^{(23)}$. Moreover, most of the negative social, psychological and physical conditions associated with worse nutritional status are partly responsive to treatment, so they should also be correctly identified and treated ${ }^{(13)}$.

The institutionalised population in Portugal is heterogeneous in terms of level of dependence, age of admission and financial situation, among other variables. Residential infrastructures for older people can be permanent or temporary; flats/houses, rooms or a combination of both; and they provide several social support activities and nursing care ${ }^{(26)}$. In 2015, the percentage of rooms was $97 \%$ out of the total offer of residential infrastructures and approximately $80 \%$ received governmental co-funding ${ }^{(26)}$. For the purposes of the present paper, the term 'nursing home' is used to refer to the overall context of residential care in Portugal. The percentage of Portuguese older adults who live in nursing homes is approximately $4 \%$, $20 \%$ of whom are independent and $20 \%$ highly dependent (bedridden or with dementia), thus determining the type of care provided ${ }^{(26,27)}$.

Facing the rapid worldwide growth in the number of older adults, it becomes more and more important to better understand and address malnutrition-associated conditions as they affect autonomy and quality of life ${ }^{(2)}$. Despite the magnitude of the problem, malnutrition awareness is still limited. It frequently goes unrecognised and therefore untreated ${ }^{(28)}$. Nationally representative Portuguese data regarding malnutrition and its associated factors in older adults living in nursing homes are missing. The current study aimed to fill this gap by: (i) characterising the nutritional status of the population aged 65 years or over living in Portuguese nursing homes; and (ii) identifying and characterising malnutrition-associated factors. The study therefore adds a national-level perspective (rather than local or regional) to the knowledge of malnutrition in the less studied nursing home setting. Furthermore, it studies the association between malnutrition and a combination of demographic, socio-economic, eating-related and psychosocial variables.

\section{Methods}

\section{Study design and participants}

Data presented herein were part of a wider study, the Portuguese Elderly Nutritional Status Surveillance System (PEN-3S). PEN-3S was a cross-sectional study of Portuguese older adults (aged 65 years or over) living in nursing homes in Portugal (mainland, Açores and Madeira 
islands). Methodological details on PEN-3S, including specific aspects of the sampling method and sample size estimations, are described elsewhere ${ }^{(29)}$.

A random cluster sampling was performed in each of the seven Portuguese regions - NUTS II (Nomenclature of Territorial Units for Statistics, as defined by the European Union). The clusters were composed of nursing homes, private or supported by the government, registered with the Portuguese Social Security Institute, which is mandatory. In each region, nursing homes were randomly listed, and were sent an invitation letter accompanied by a full written explanation of the study objectives and methods. Institutions were invited to enrol in subsequent order until the target number of residents, according to sample size estimations, was achieved in each region.

Exclusion criteria were: individuals living in Portugal for less than 1 year; non-Portuguese speakers; absence from the institution during data collection (e.g. due to hospitalisation); those who could not understand and/or answer the questionnaire due to dementia, psychiatric disease or severe hearing and/or visual impairment; and bedridden individuals. All eligible residents aged 65 years or over living in participating nursing homes were invited face-toface by the interviewer to participate in the study. In cases of acceptance to participate, the interview was conducted in the next few days.

The research protocol followed the Helsinki Declaration $^{(30)}$. Permissions from the National Data Protection Commission and the Faculdade de Medicina, Universidade de Lisboa Ethics Commission were obtained. All volunteer participants signed a written informed consent prior to the beginning of the study ${ }^{(29)}$.

\section{Data collection}

Data were collected through a computer-assisted face-toface structured interview followed by anthropometric measurements, conducted by seven trained nutritionists, as described elsewhere ${ }^{(29)}$. Data were collected between October 2015 and April 2016.

Demographic and socio-economic data, including age, sex, educational level, marital status, self-perception of financial situation and total time living in the nursing home, as well as data on self-reported morbidity, self-rated appetite in the last $7 \mathrm{~d}$, eating difficulties due to oral health problems in the last $7 \mathrm{~d}$, nutritional status, anthropometry, cognitive function, symptoms of depression, loneliness feelings and functional status, were collected. For demographic, socio-economic, behavioural and morbidity data, the National Food, Nutrition and Physical Activity Survey (IAN-AF) methodology was used and data were collected through the platform 'YOU, eAT\& MOVE', developed by $\mathrm{IAN}-\mathrm{AF}^{(31)}$.

Single-item measures were used to assess selfperception of financial situation ( $1=$ 'very comfortable' to $5=$ 'very hard'), self-rated appetite in the last $7 \mathrm{~d}(1=$ 'no appetite' to $5=$ 'lots of appetite') and eating difficulties due to oral health problems in the last $7 \mathrm{~d}(1=$ 'many' to $4=$ 'none').

\section{Cognitive function}

The thirty-item Mini Mental State Examination (MMSE) was used to assess global cognitive function. Questions are grouped into seven categories: (i) temporal and spatial orientation; (ii) working memory; (iii) recall; (iv) attention; (v) arithmetic capacity; (vi) linguistic skills; and (vii) visual-motor skills. The maximum score is 30 points; the minimum cut-offs for inadequate cognitive functioning used in the present study were those validated for the Portuguese population according to the number of years attending school: 0 years, $\leq 15$ points; $1-11$ years, $\leq 22$ points; $>11$ years, $\leq 27$ points. In the present study, MMSE score determined if the individual would answer all questionnaires $^{(32,33)}$. If MMSE score was lower than the cut-off, the data collected regarded only sociodemographic data, self-rated appetite, self-reported eatingrelated difficulties and nutritional status (anthropometric measures and $\mathrm{MNA}^{\circledR}$ ).

\section{Nutritional status}

Nutritional status was assessed with the eighteen-item MNA ${ }^{\circledR(34-36)}$. The full $\mathrm{MNA}^{\circledR}$, hereinafter referred to as MNA $^{\circledR}$, is organised into four parts: (i) anthropometry; (ii) dietary assessment; (iii) self-perception of nutritional status and health; and (iv) a general evaluation of lifestyle, medication, depression and dementia. The total score ranges from 0 to 30 points, classifying individuals as normal (24-30 points), at risk of malnutrition (17-23.5 points) or malnourished ( $<17$ points $)^{(28)}$. This is a valid instrument showing a high association with objective indicators of nutritional status (e.g. triceps skinfold, energy intake, serum albumin) and clinical assessment of nutritional status $^{(35)}$. Low MNA ${ }^{\circledR}$ scores have been shown to predict worse clinical outcomes and higher mortality ${ }^{(35)}$.

\section{Anthropometry}

Anthropometric measurements followed the International Standards for Anthropometric Assessment of the International Society for the Advancement of Kinanthropometry, with individuals wearing minimal clothing and no shoes ${ }^{(37)}$. Weight was measured to the nearest $0.1 \mathrm{~kg}$ with a portable calibrated scale (SECA Robusta $813^{\circledR}$ ). Whenever possible, height was measured using a portable stadiometer to the nearest $0 \cdot 1 \mathrm{~cm}$ (SECA $214^{\circledR}$ ); if not, hand length was measured with a fibreglass tape to the nearest $0 \cdot 1 \mathrm{~cm}$ and used to estimate height with the validated equations for the Portuguese population ${ }^{(38)}$. Arm and calf circumferences were measured to the nearest $0 \cdot 1 \mathrm{~cm}$ using a non-extensible, flexible, fibreglass tape (SECA $201^{\circledR}$ ).

BMI was defined as weight in kilograms divided by the square of height in metres $\left(\mathrm{kg} / \mathrm{m}^{2}\right)$. If no BMI could be calculated, the MNA ${ }^{\circledR}$-Short Form $\left(\mathrm{MNA}^{\circledR}-\mathrm{SF}\right)$ version that 
uses calf circumference was applied instead ${ }^{(39)}$. The WHO cut-offs $\quad\left(\mathrm{BMI}<18.5 \mathrm{~kg} / \mathrm{m}^{2} ; \quad 18.5 \leq \mathrm{BMI}<25.0 \mathrm{~kg} / \mathrm{m}^{2}\right.$; $25.0 \leq \mathrm{BMI}<30 \cdot 0 \mathrm{~kg} / \mathrm{m}^{2} ; \mathrm{BMI} \geq 30 \cdot 0 \mathrm{~kg} / \mathrm{m}^{2}$ ) were used to categorise individuals according to their BMI when this was presented as a stand-alone measure ${ }^{(15,40)}$. It is noteworthy that the BMI item in MNA ${ }^{\circledR}$ (item F) was scored according to MNA instructions $\left(<19.0 \mathrm{~kg} / \mathrm{m}^{2}, 0\right.$ points; $19 \cdot 0-20 \cdot 9 \mathrm{~kg} / \mathrm{m}^{2}, \quad 1$ point; $21 \cdot 0-22 \cdot 9 \mathrm{~kg} / \mathrm{m}^{2}, \quad 2$ points; $\geq 23.0 \mathrm{~kg} / \mathrm{m}^{2}, 3$ points), thus not following WHO criteria.

\section{Symptoms of depression}

The fifteen-item version of the Geriatric Depression Scale (GDS-15) was used to screen for depression symptomatology ${ }^{(41)}$. We used a cut-off of 5 to categorise respondents as presenting symptoms of depression (score $>5$ ) or $\operatorname{not}^{(42)}$.

Functional status: instrumental activities of daily living The eight-item Lawton scale was used to measure the instrumental daily living activities (IADL). Each item measured by the Lawton scale relies on either cognitive or physical function, although all require some degree of both. Scores range from 0 (low function, dependent) to 8 (high function, independent), and a cut-off of 8 was used to classify individuals as non-dependent ${ }^{(43)}$.

\section{Loneliness}

The sixteen-item UCLA Loneliness Scale was used to measure participants' subjective feelings of loneliness and social isolation. Scores range between 16 and 64 points, with higher values indicating higher subjective feeling of loneliness ${ }^{(44)}$. We used the proposed cut-off for the Portuguese population: loneliness feelings if the score is $>32^{(45)}$.

\section{Statistical methods}

Statistical analyses were performed using the statistical software package IBM SPSS Statistics ${ }^{\circledR}$ version 24 and the statistical significance level was set to $5 \%$. Data were weighted according to the distribution of the Portuguese population living in nursing homes in each of the seven NUTS II. These weighting corrections and the design effect were adjusted when running statistical procedures (both descriptive and inferential) through complex sample analysis, except for the demographic and socio-economic characterisation for which non-weighted data were used. The normality of the distributions was assessed through the Kolmogorov-Smirnov test, taking into consideration kurtosis values and visual inspection of QQ plots. Between-group differences were assessed using the independent-samples $t$ test or Mann-Whitney $U$ test (continuous variables), the $\chi^{2}$ test (categorical variables), and ANOVA or the non-parametric Kruskal-Wallis test (more than two groups). To compare two continuous variables, correlation coefficients $(r)$ were calculated using simple correlations after weighting the sample by NUTS II. To identify malnutrition-associated factors at the population level, bivariate and logistic regression complex sample analyses were used to evaluate the associations between malnutrition status (dependent variable, composed of individuals who were malnourished or at risk of malnutrition) and the different variables in study. Clinically relevant covariates with $P \leq 0 \cdot 10$ in the bivariate analysis and with explanatory power were included in the logistic regression. Model 1 was unadjusted; model 2 was further adjusted for sex, age and financial situation; and in model 3 all studied variables were adjusted for each other, except for educational level and marital status to avoid possible collinearity.

\section{Results}

\section{Sample characterisation, demographic and socio-} economic characteristics

Participants were recruited from the seven Portuguese NUTS II, in a total of seventy-nine randomly selected nursing homes that accepted to participate (participation rate $=62 \%$ ): nine from Norte region, twelve from Centro, thirteen from Área Metropolitana de Lisboa, eleven from Alentejo, ten from Algarve, fourteen from Madeira and ten from Açores. The vast majority of nursing homes were non-profit organisations, not administered by the government but receiving governmental co-funding. The mean number of residents was $61 \cdot 3$ (sD 46.1).

In total, 1275 individuals were invited to participate in the present study, 1186 of whom accepted to enrol (93\%). The mean number of participating residents from each nursing home was 15 (SD 9.2; range $=2-40$ ). Participants and refusals did not differ by sex (72.8 v. 68.5\% women), mean age ( $83.4 v .82 .5$ years old), educational level ( 89.2 $v .88 .7 \%$ with $<5$ years of formal education) and presence of diseases requiring regular health care (65.8 v. 66.0\%).

Demographic and socio-economic characteristics of the sample are presented in Table 1. Eight hundred and sixtyfour $(72.8 \%)$ participants were women, and mean age was $83.4(95 \%$ CI $82 \cdot 8,83 \cdot 7)$ years. Almost half of the sample $(49.2 \%)$ was aged $\geq 85$ years. Regarding marital status, $69.7 \%$ of women were widowed $v .50 .9 \%$ of men. Eightynine per cent of the sample did not go to school or only attended primary school ( $<5$ years), and $37.4 \%$ considered their financial situation as hard or very hard. The mean length of time living in the current nursing home was 51.7 months (i.e. 4 years and 4 months; $95 \%$ CI 47.9 , 55.5 months) and $29.8 \%$ reported living there for $>5$ years.

Complex sample analysis revealed that 42.7 (95\% CI $38 \cdot 5,46.9) \%$ reported their appetite as ' 3 ' and $24.2 \%$ as ' 1 ' or ' 2 ' on a scale from 1 ('no appetite') to 5 ('lots of appetite'). Also, 59.5 (95\% CI 55.4, 63.4)\% reported no eating difficulties due to oral health problems, while $31.5 \%$ reported some/many eating difficulties. Table 2 
Table 1 Demographic and socio-economic characteristics of participants: a nationally representative sample of nursing home residents aged 65 years or over in Portugal, October 2015-April 2016

\begin{tabular}{|c|c|c|c|c|c|c|}
\hline & \multicolumn{2}{|c|}{ Women } & \multicolumn{2}{|c|}{ Men } & \multicolumn{2}{|c|}{ Total } \\
\hline & $\%$ & $n$ & $\%$ & $n$ & $\%$ & $n$ \\
\hline Total & $72 \cdot 8$ & 864 & $27 \cdot 2$ & 322 & 100 & 1186 \\
\hline \multicolumn{7}{|l|}{ Age (years) } \\
\hline$<75$ & $10 \cdot 2$ & 88 & $17 \cdot 1$ & 55 & $12 \cdot 1$ & 143 \\
\hline $75-84$ & 38.7 & 334 & 38.8 & 125 & 38.7 & 459 \\
\hline$\geq 85$ & $51 \cdot 2$ & 442 & $44 \cdot 1$ & 142 & $49 \cdot 2$ & 584 \\
\hline \multicolumn{7}{|l|}{ Marital status } \\
\hline Single & $18 \cdot 4$ & 159 & 16.5 & 53 & $17 \cdot 9$ & 212 \\
\hline Divorced & 3.4 & 29 & 8.4 & 27 & 4.7 & 56 \\
\hline Widowed & 69.7 & 602 & $50 \cdot 9$ & 164 & 64.6 & 766 \\
\hline Married or living together & 8.6 & 74 & $24 \cdot 2$ & 78 & $12 \cdot 8$ & 152 \\
\hline \multicolumn{7}{|l|}{ Education (years) } \\
\hline$<5$ & 89.6 & 774 & $87 \cdot 3$ & 281 & 89.0 & 1055 \\
\hline $5-9$ & $6 \cdot 4$ & 55 & 7.5 & 24 & $6 \cdot 7$ & 79 \\
\hline$>9$ & $4 \cdot 1$ & 35 & $5 \cdot 3$ & 17 & 4.4 & 52 \\
\hline \multicolumn{7}{|l|}{ Financial situation } \\
\hline Very comfortable & 0.7 & 6 & 0.0 & 0 & 0.5 & 6 \\
\hline Comfortable & $6 \cdot 4$ & 55 & 9.0 & 29 & $7 \cdot 1$ & 84 \\
\hline Enough for my needs & 30.5 & 263 & 42.5 & 137 & 33.8 & 400 \\
\hline Hard & $28 \cdot 0$ & 241 & 24.8 & 80 & $27 \cdot 1$ & 321 \\
\hline Very hard & $10 \cdot 7$ & 92 & $9 \cdot 3$ & 30 & $10 \cdot 3$ & 122 \\
\hline Did not know/did not answer & $23 \cdot 8$ & 205 & $14 \cdot 3$ & 46 & $21 \cdot 2$ & 251 \\
\hline \multicolumn{7}{|c|}{ Total time in nursing home (months) $\dagger$} \\
\hline$<12$ & $17 \cdot 2$ & 119 & $23 \cdot 1$ & 63 & $18 \cdot 9$ & 182 \\
\hline $12-23$ & $18 \cdot 1$ & 125 & $15 \cdot 0$ & 41 & $17 \cdot 2$ & 166 \\
\hline $24-59$ & $34 \cdot 3$ & 237 & 33.7 & 92 & 34.1 & 329 \\
\hline$>60$ & 30.4 & 210 & $28 \cdot 2$ & 77 & $29 \cdot 8$ & 287 \\
\hline
\end{tabular}

†For 'Total time in nursing home (months)', $n 964$.

Table 2 Eating-related difficulties and other clinical conditions among nursing home residents aged 65 years or over ( $n$ 1186) in Portugal, October 2015-April 2016

\begin{tabular}{|c|c|c|c|c|c|c|c|}
\hline & \multicolumn{2}{|c|}{ Women } & \multicolumn{2}{|c|}{ Men } & \multicolumn{2}{|c|}{ Total } & \multirow[b]{2}{*}{$P \dagger$} \\
\hline & $\%$ & $95 \% \mathrm{Cl}$ & $\%$ & $95 \% \mathrm{Cl}$ & $\%$ & $95 \% \mathrm{Cl}$ & \\
\hline \multicolumn{8}{|l|}{ Appetite $(n 1184)$} \\
\hline 1 ('no appetite') & $7 \cdot 1$ & $4 \cdot 7,10 \cdot 6$ & $2 \cdot 9$ & $1 \cdot 2,6 \cdot 7$ & 5.8 & $3.9,8.6$ & 0.070 \\
\hline 2 & $20 \cdot 3$ & $17 \cdot 0,24 \cdot 0$ & $14 \cdot 0$ & $9 \cdot 4,20 \cdot 4$ & 18.4 & $15 \cdot 9,21 \cdot 2$ & \\
\hline 3 & $41 \cdot 6$ & $36 \cdot 4,47 \cdot 0$ & $45 \cdot 1$ & $38 \cdot 2,52 \cdot 2$ & $42 \cdot 7$ & $38.5,46.9$ & \\
\hline 4 & 21.5 & $16 \cdot 9,26 \cdot 9$ & $25 \cdot 1$ & $21 \cdot 5,29 \cdot 1$ & $22 \cdot 6$ & $19 \cdot 0,26 \cdot 6$ & \\
\hline 5 ('lots of appetite') & 9.5 & $6 \cdot 8,13 \cdot 1$ & $12 \cdot 9$ & $9 \cdot 0,18 \cdot 1$ & 10.5 & $8 \cdot 3,13 \cdot 3$ & \\
\hline \multicolumn{8}{|c|}{ Eating difficulties due to oral health problems ( $n$ 1184) } \\
\hline Many & $14 \cdot 1$ & $10 \cdot 9,18 \cdot 2$ & 11.5 & $7 \cdot 1,18 \cdot 1$ & $13 \cdot 3$ & $10 \cdot 3,17 \cdot 0$ & 0.211 \\
\hline Some & $19 \cdot 8$ & $15 \cdot 9,24 \cdot 4$ & 14.5 & $10 \cdot 7,19.2$ & $18 \cdot 2$ & $15 \cdot 3,21 \cdot 4$ & \\
\hline Few & $9 \cdot 1$ & $6 \cdot 4,12 \cdot 9$ & 8.0 & $5 \cdot 2,12 \cdot 1$ & 8.8 & $6 \cdot 6,11.6$ & \\
\hline None & $56 \cdot 8$ & $52 \cdot 3,61 \cdot 3$ & 65.5 & $57 \cdot 9,72 \cdot 4$ & 59.5 & $55 \cdot 4,63.4$ & \\
\hline \multicolumn{8}{|c|}{ Having diseases that need regular health care $(n 576)$} \\
\hline Yes & $79 \cdot 8$ & $71 \cdot 1,86 \cdot 4$ & $77 \cdot 0$ & $66 \cdot 3,85 \cdot 0$ & 78.9 & $71 \cdot 7,84 \cdot 7$ & 0.598 \\
\hline \multicolumn{8}{|l|}{ Clinical diagnosis } \\
\hline Cardiac disease ( $n$ 551) & $45 \cdot 0$ & $38 \cdot 6,51 \cdot 6$ & $37 \cdot 4$ & $29 \cdot 3,46 \cdot 4$ & $42 \cdot 5$ & $37 \cdot 4,47 \cdot 8$ & 0.167 \\
\hline Type 2 diabetes ( $n$ 562) & 29.8 & $24 \cdot 8,35 \cdot 5$ & $35 \cdot 3$ & $26 \cdot 1,45 \cdot 8$ & 31.6 & $25 \cdot 9,38.0$ & 0.177 \\
\hline Hypertension ( $n$ 541) & $68 \cdot 1$ & $60 \cdot 5,74.8$ & $64 \cdot 2$ & $57 \cdot 3,70 \cdot 5$ & $66 \cdot 8$ & $61 \cdot 2,72 \cdot 1$ & 0.411 \\
\hline Dyslipidaemia ( $n$ 513) & $50 \cdot 3$ & $43 \cdot 1,57 \cdot 6$ & $46 \cdot 8$ & $38.7,55 \cdot 0$ & $49 \cdot 2$ & $43 \cdot 1,55 \cdot 3$ & 0.471 \\
\hline Gastrointestinal disease (n 539) & $37 \cdot 3$ & $29 \cdot 8,45 \cdot 5$ & $22 \cdot 6$ & $15 \cdot 1,32 \cdot 5$ & $32 . \overline{6}$ & $26 \cdot 7,39 \cdot 2$ & 0.019 \\
\hline Arthritis ( $n 501)$ & 40.9 & $30 \cdot 1,52 \cdot 7$ & 23.0 & $11.9,39.7$ & $35 \cdot 1$ & $25 \cdot 1,46 \cdot 8$ & 0.013 \\
\hline Osteoporosis (n 503) & 31.6 & $24 \cdot 9,39 \cdot 2$ & 5.6 & $2 \cdot 4,12 \cdot 6$ & $22 \cdot 8$ & $17 \cdot 9,28.5$ & $<0.001$ \\
\hline \multicolumn{8}{|l|}{ Cognitive function ( $n$ 1185) } \\
\hline Impaired according to MMSE & 47.5 & $41 \cdot 5,53 \cdot 6$ & $41 \cdot 6$ & $34 \cdot 2,49 \cdot 3$ & $45 \cdot 7$ & $40 \cdot 1,51 \cdot 4$ & 0.131 \\
\hline
\end{tabular}

MMSE, Mini Mental State Examination.

† Second-order Rao-Scott adjusted $X^{2}$ statistic to compare between women and men. Significance based on adjusted $F$ and degrees of freedom (complex sample analysis). 
shows frequencies for several self-reported chronic diseases. For example, $29 \cdot 8$ (95\% CI $24 \cdot 8,35 \cdot 5) \%$ of women and 35.3 (95\% CI $26 \cdot 1,45.8) \%$ of men reported clinical diagnosis of type 2 diabetes. Gastrointestinal diseases, arthritis and osteoporosis diagnosis were significantly more frequent among women than men. Overall, 78.9 (95\% CI 71.7, 84.7)\% reported having a disease requiring regular health care (e.g. medical appointments and examinations).

\section{Nutritional status and anthropometry}

$\mathrm{MNA}^{\circledR}$ score was calculated for all participants. MNA ${ }^{\circledR}$-SF was used instead of the eighteen-item MNA ${ }^{\circledR}$ for 144 participants due to missing weight data (results for participants with missing weight are presented under 'Nutritional status-associated factors'). According to MNA ${ }^{\circledR}, 4 \cdot 8$ (95\% CI 3.2, 7.3) \% were classified as malnourished and 38.7 (95\% CI 33.5, 44.2)\% at risk of malnutrition. These percentages were higher for women than men: $5.3(95 \%$ CI $3.3,8.3) \%$ v. $3.9(95 \%$ CI $1.9,7.5) \%$ were malnourished, and 43.6 (95\% CI 38.1, 49.2) \% v. $27.5(95 \% \mathrm{CI}$ $20 \cdot 6,35.6) \%$ were at risk of malnutrition, respectively (Table 3). The percentage of individuals at risk of malnutrition was significantly higher among those in the upper age group. When taking the educational level into account, risk of malnutrition was more frequent among those who attended school for $<5$ years. Considering BMI and $\mathrm{MNA}^{\circledR}$ categories simultaneously, 52.9 (95\% CI 44.0, $61.7) \%$ of those classified as normal weight $\left(18.5 \leq \mathrm{BMI}<25.0 \mathrm{~kg} / \mathrm{m}^{2}\right)$ were at risk of malnutrition according to MNA ${ }^{\circledR}$. Of those in the pre-obesity category $\left(25 \cdot 0 \leq \mathrm{BMI}<30.0 \mathrm{~kg} / \mathrm{m}^{2}\right), 27 \cdot 6(95 \%$ CI $21 \cdot 4,34 \cdot 7) \%$ were at risk of malnutrition and 1.4 (95\% CI $0.4,4.5) \%$ were malnourished.

Mean BMI was $27 \cdot 4(95 \%$ CI $27 \cdot 0,27 \cdot 8) \mathrm{kg} / \mathrm{m}^{2}$ and it was significantly higher for women $(P<0 \cdot 001)$, decreasing with age $(r=-0 \cdot 18, P<0 \cdot 001)$ and with education $(r=-0 \cdot 10$, $P=0.001)$. Using WHO cut-offs, $3.3(95 \%$ CI $1.9,5 \cdot 7) \%$ were classified as underweight, $27 \cdot 5$ (95\% CI 25.0, 30.2)\% as normal weight, $40.5(95 \% \mathrm{CI} 36 \cdot 2,45.0) \%$ as pre-obese and $28 \cdot 6$ (95\% CI $24 \cdot 8,32 \cdot 8) \%$ as obese (Fig. 1).

\section{Cognitive function, symptoms of depression and} functional status

According to MMSE cut-offs, 52.5 (95\% CI $46 \cdot 4,58 \cdot 5) \%$ of women and $58.4(95 \%$ CI $50 \cdot 7,65 \cdot 8) \%$ of men had a nonimpaired cognitive function and therefore were considered eligible to answer the full interview. The mean MMSE total score was significantly higher for men $($ mean $=21.9,95 \%$ CI $21 \cdot 0,22 \cdot 8)$ than women $($ mean $=$ $19 \cdot 8,95 \%$ CI $18 \cdot 8,20 \cdot 8 ; P<0 \cdot 001)$.

The mean GDS-15 total score was $6 \cdot 1$ (95\% CI 5.6, 6.5) and it was significantly higher for women $(P<0.001)$. Using a score of 5 as cut-off, $60 \cdot 4(95 \%$ CI $52 \cdot 9,67 \cdot 4) \%$ of women and $37.2(95 \%$ CI $28.0,47.4) \%$ of men had symptoms of depression.

Mean score of the Lawton scale was $4.6(95 \%$ CI $4 \cdot 3$, $4 \cdot 8)$, corresponding to the 'moderate dependence' category. According to Lawton cut-offs, $11 \cdot 0$ (CI 95\% 8.0, $14.8) \%$ were considered totally dependent, 25.3 (95\% CI $20 \cdot 8,30 \cdot 5) \%$ severely dependent and 12.9 (95\% CI 9.2, 17.9) \% independent.

Table 3 Distribution of sociodemographic characteristics and BMI (WHO criteria) according to nutritional status category (MNA ${ }^{\circledR}$ ) among nursing home residents aged 65 years or over $(n$ 1186) in Portugal, October 2015-April 2016

\begin{tabular}{|c|c|c|c|c|c|c|c|}
\hline & \multicolumn{2}{|c|}{ Normal nutritional status } & \multicolumn{2}{|c|}{ At risk of malnutrition } & \multicolumn{2}{|c|}{ Malnourished } & \multirow[b]{2}{*}{$P+$} \\
\hline & $\%$ & $95 \% \mathrm{Cl}$ & $\%$ & $95 \% \mathrm{Cl}$ & $\%$ & $95 \% \mathrm{Cl}$ & \\
\hline \multicolumn{8}{|l|}{ Sex } \\
\hline Women & $51 \cdot 2$ & $45 \cdot 3,57 \cdot 0$ & $43 \cdot 6$ & $38 \cdot 1,49 \cdot 2$ & $5 \cdot 3$ & $3 \cdot 3,8 \cdot 3$ & \multirow[t]{3}{*}{$<0.001$} \\
\hline Men & $68 \cdot 7$ & $60 \cdot 5,75 \cdot 8$ & 27.5 & $20 \cdot 6,35 \cdot 6$ & 3.9 & $1.9,7.5$ & \\
\hline Total & $56 \cdot 5$ & $50 \cdot 8,62 \cdot 0$ & 38.7 & $33 \cdot 5,44 \cdot 2$ & 4.8 & $3.2,7.3$ & \\
\hline \multicolumn{8}{|l|}{ Age (years) } \\
\hline$<75$ & 68.9 & $54.5,80 \cdot 3$ & $27 \cdot 3$ & $16 \cdot 9,41 \cdot 1$ & 3.8 & $1 \cdot 1,12 \cdot 0$ & \multirow{4}{*}{0.026} \\
\hline $75-84$ & $60 \cdot 6$ & $52 \cdot 0,68 \cdot 6$ & 33.5 & $26 \cdot 3,41 \cdot 5$ & 5.9 & $3 \cdot 3,10 \cdot 2$ & \\
\hline$\geq 85$ & 51.6 & $46 \cdot 1,57 \cdot 1$ & $44 \cdot 1$ & $38.5,49.9$ & 4.3 & $2 \cdot 3,7 \cdot 6$ & \\
\hline Total & $56 \cdot 5$ & $50 \cdot 8,62 \cdot 0$ & 38.7 & $33 \cdot 5,44 \cdot 2$ & 4.8 & $3.2,7.3$ & \\
\hline \multicolumn{8}{|c|}{ Education (years) } \\
\hline$<5$ & 55.0 & $48 \cdot 6,61 \cdot 2$ & 39.9 & $34 \cdot 1,45 \cdot 9$ & $5 \cdot 1$ & $3.3,7.9$ & \multirow{4}{*}{0.222} \\
\hline $5-9$ & $70 \cdot 3$ & $57 \cdot 1,80 \cdot 8$ & $27 \cdot 0$ & $17.6,39.0$ & $2 \cdot 7$ & $0.4,17 \cdot 3$ & \\
\hline$>9$ & 63.9 & $50 \cdot 3,75 \cdot 6$ & 33.8 & $22 \cdot 2,47 \cdot 7$ & $2 \cdot 3$ & $0.3,14.4$ & \\
\hline Total & $56 \cdot 5$ & $50 \cdot 8,62 \cdot 0$ & 38.7 & $33 \cdot 5,44 \cdot 2$ & 4.8 & $3 \cdot 2,7 \cdot 3$ & \\
\hline \multicolumn{8}{|l|}{ BMI $\left(\mathrm{kg} / \mathrm{m}^{2}\right)$} \\
\hline$<18.5$ & $12 \cdot 9$ & $3 \cdot 4,38 \cdot 1$ & 48.0 & $23 \cdot 2,73 \cdot 9$ & 39.0 & $13 \cdot 4,72 \cdot 6$ & \multirow[t]{4}{*}{$<0.001$} \\
\hline $18.5-24.9$ & $43 \cdot 4$ & $34 \cdot 2,53 \cdot 2$ & $52 \cdot 9$ & $44 \cdot 0,61 \cdot 7$ & 3.6 & $1.3,9.9$ & \\
\hline $25 \cdot 0-29 \cdot 9$ & $71 \cdot 1$ & $63 \cdot 8,77 \cdot 4$ & $27 \cdot 6$ & $21 \cdot 4,34 \cdot 7$ & 1.4 & $0.4,4.5$ & \\
\hline$\geq 30.0$ & 68.0 & $61 \cdot 0,74 \cdot 3$ & 31.7 & $25 \cdot 5,38.6$ & 0.3 & $0.0,2.4$ & \\
\hline
\end{tabular}

MNA $^{\circledR}$, Mini Nutritional Assessment.

† Second-order Rao-Scott adjusted $X^{2}$ statistic to compare between MNA ${ }^{\circledR}$ categories. Significance based on adjusted $F$ and degrees of freedom (complex sample analysis). 
(a)

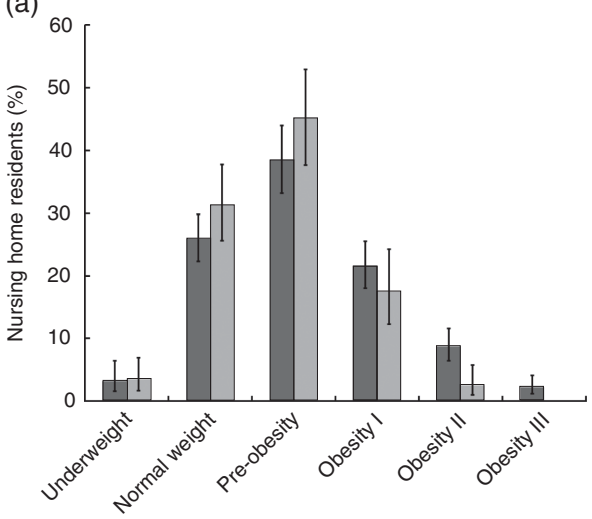

BMI category (b)

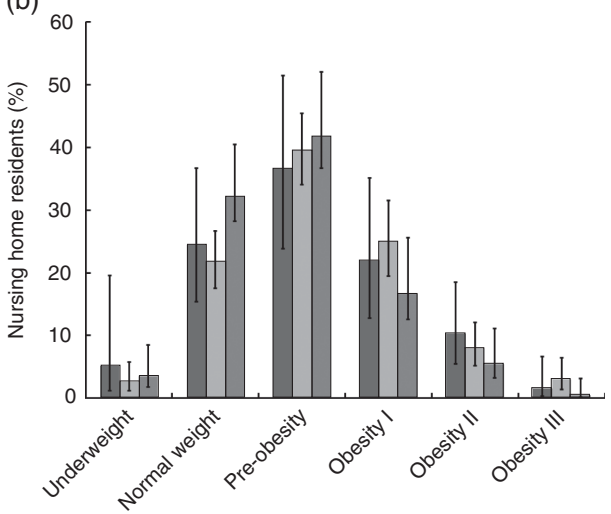

BMI category

Fig. 1 Distribution of participants by BMI category according to WHO criteria†: (a) distribution by sex ( $\square$, women; $\square$, men) and (b) distribution by age ( $\square,<75$ years old; $\square, 75-84$ years old; $\square, \geq 85$ years old) among nursing home residents aged 65 years or over ( $n$ 1186) in Portugal, October 2015-April 2016. Values are means with their $95 \% \mathrm{Cl}$ represented by vertical bars. †Underweight, $\mathrm{BMl}<18.5 \mathrm{~kg} / \mathrm{m}^{2}$; normal weight, $18.5 \leq \mathrm{BMl}<25.0 \mathrm{~kg} / \mathrm{m}^{2}$; pre-obese, $25.0 \leq \mathrm{BMl}<30.0 \mathrm{~kg} / \mathrm{m}^{2}$; obese I, $30.0 \leq \mathrm{BMl}<34.9 \mathrm{~kg} / \mathrm{m}^{2}$; obese II, $35.0 \leq \mathrm{BMI}<39.9 \mathrm{~kg} / \mathrm{m}^{2}$; obese III, BMI $\geq 40.0 \mathrm{~kg} / \mathrm{m}^{2}$

The mean total score for the UCLA Loneliness Scale was 32.3 (95\% CI 31.1, 33.5). Applying this scale's cut-off point, 42.5 (95\% CI 37.3, 47.9) \% reported loneliness feelings, this percentage being $44.4 \%$ for women and $38.6 \%$ for men, without significant differences between these two groups.

\section{Nutritional status-associated factors}

Bivariate analysis showed that $\operatorname{sex}(P<0 \cdot 001)$, age group $(P=0.019)$, educational level $(P=0 \cdot 024)$, marital status $(P=0.046)$, financial situation $(P<0.001)$, appetite $(P<0.001)$ and eating difficulties due to oral health problems $(P<0.001)$ were significantly associated with nutritional status (being at risk of malnutrition or being malnourished $v$. normal nutritional status; see online supplementary material, Supplemental Table 1). Impaired cognitive function $(P<0.001)$, symptoms of depression $(P<0.001)$, worse functional status to perform IADL $(P<0.001)$ and loneliness feelings $(P=0.017)$ were also significantly associated with malnutrition risk/malnutrition.

As previously mentioned, it was not possible to measure weight for 144 participants and hand length was used to estimate height for the majority of participants ( $n$ 887). Those who were not weighed had more eating difficulties due to oral health problems (50.5 v. 29.8\%, $P=0.002)$, were more frequently cognitively impaired (64.3 v. 44.0\%, $P<0.001)$, dependent $(99.5$ v. $86.4 \%$, $P<0.001)$ and malnourished $(25.2$ v. $2.9 \%, P<0.001)$. Individuals whose height was not measured were older (84.8 v. 82.0 years old, $P=0.002$ ), and more frequently reported poor or no appetite $(26.8 v .15 \cdot 0 \%, P=0.023)$, were cognitively impaired ( $48.5 v .35 \cdot 6 \%, P=0.010)$ and at risk of malnutrition or malnourished (48.3 v. $26.3 \%$, $P<0 \cdot 001)$.

After adjusting for all studied potential confounders (model 3), loneliness feelings no longer significantly increased the odds of being malnourished (cf. model 2). Results from the adjusted model 3 showed that the OR for being at risk of malnutrition or being malnourished decreased with increasing appetite, 6.5 (95\% CI 2.7, 15.3) for those who self-rated appetite as ' 1 ' ('no appetite') or ' 2 ', and 2.6 (95\% CI $1 \cdot 2,5 \cdot 5)$ for people rating appetite as ' 3 ', compared with those who considered their appetite as ' 4 ' or ' 5 ('lots of appetite'; Table 4). Individuals who reported symptoms of depression (GDS-15 score $>5$ ) had an OR for malnutrition risk/malnutrition of 2.6 (95\% CI 1.6, 4.2) compared with non-depressed older adults, and individuals who were classified as dependent (Lawton scale) had an OR of 4.7 (95\% CI $2 \cdot 0,11 \cdot 1)$ compared with independent older adults. All other studied variables were not significantly associated with malnutrition in the final adjusted model (model 3).

\section{Discussion}

The present study is the first including a Portuguese nationally representative sample of nursing home residents aged 65 years or over, not bedridden nor with diagnosed dementia. The prevalence of malnutrition among older adults residing in nursing homes was $4.8 \%$ and of risk of malnutrition was $38.7 \%$. The study also found that poor appetite, depression symptomatology and lower IADL functionality independently increased the odds of being malnourished or at risk of malnutrition.

Although sex and age distributions of the collected sample are in line with published international studies, marital status and educational level distributions are different ${ }^{(46,47)}$. For example, the percentage of widowed participants $(64.6 v .53 \%)$ and of those who only attended primary school $(89.0 \mathrm{v}$. 61\%) were higher in our study than in a Finnish sample of older residents ${ }^{(47)}$. To our 
Table 4 OR for likelihood of being at risk of malnutrition or malnourished according to demographic and socio-economic characteristics, appetite, eating-related difficulties and psychosocial variables among nursing home residents aged 65 years or over without cognitive impairment ( $n$ 522) in Portugal, October 2015-April 2016†,‡,§

\begin{tabular}{|c|c|c|c|c|c|c|}
\hline & \multicolumn{2}{|c|}{ Model 1} & \multicolumn{2}{|c|}{ Model 2} & \multicolumn{2}{|c|}{ Model 3} \\
\hline & OR & $95 \% \mathrm{Cl}$ & OR & $95 \% \mathrm{Cl}$ & OR & $95 \% \mathrm{Cl}$ \\
\hline \multicolumn{7}{|l|}{ Sex } \\
\hline Men & 1.00 & Ref. & 1.00 & Ref. & 1.00 & Ref. \\
\hline Women & $1 \cdot 74^{\star}$ & $1.05,2.90$ & 1.45 & $0.86,2.44$ & $1 \cdot 17$ & $0.67,2.06$ \\
\hline \multicolumn{7}{|l|}{ Age (years) } \\
\hline$<75$ & 1.00 & Ref. & 1.00 & Ref. & 1.00 & Ref. \\
\hline $75-84$ & 0.95 & $0.43,2.13$ & 0.85 & $0.33,2.19$ & 0.88 & $0.30,2.60$ \\
\hline$\geq 85$ & 1.55 & $0.68,3.58$ & 1.33 & $0.51,3.46$ & 0.95 & $0.34,2.65$ \\
\hline \multicolumn{7}{|l|}{ Marital status } \\
\hline Married or living together & 1.00 & Ref. & 1.00 & Ref. & 1.00 & Ref. \\
\hline Single & 1.49 & $0.58,3.84$ & 1.22 & $0.45,3.32$ & 1.62 & $0.62,4.24$ \\
\hline Divorced or widowed & 2.04 & $0.93,4.48$ & 1.60 & $0.77,3.34$ & 1.77 & $0.79,3.98$ \\
\hline \multicolumn{7}{|l|}{ Education (years)\| } \\
\hline$<5$ & 1.00 & Ref. & 1.00 & Ref. & 1.00 & Ref. \\
\hline$\geq 5$ & 0.62 & $0.29,1.35$ & 0.62 & $0.27,1.41$ & 0.60 & $0.25,1.46$ \\
\hline \multicolumn{7}{|l|}{ Financial situation } \\
\hline Very comfortable or comfortable & 1.00 & Ref. & 1.00 & Ref. & 1.00 & Ref. \\
\hline Enough for my needs & 1.23 & $0.47,3.19$ & $1 \cdot 16$ & $0.43,3.14$ & 1.28 & $0.40,4.06$ \\
\hline Hard or very hard & $3 \cdot 16^{\star}$ & $1.36,7.38$ & $2 \cdot 84^{*}$ & $1.14,7.08$ & 2.52 & $0.83,7.64$ \\
\hline \multicolumn{7}{|l|}{ Appetite } \\
\hline 1 ('no appetite') or 2 & $7 \cdot 80^{\star}$ & $3 \cdot 35,18 \cdot 16$ & $7 \cdot 79^{*}$ & $3.54,17.14$ & $6 \cdot 46^{*}$ & $2 \cdot 72,15 \cdot 34$ \\
\hline 3 & $2 \cdot 41^{*}$ & $1 \cdot 26,4.61$ & $2 \cdot 65^{\star}$ & $1.38,5.09$ & $2 \cdot 61^{*}$ & $1.23,5.53$ \\
\hline 4 or 5 ('lots of appetite') & 1.00 & Ref. & 1.00 & Ref. & 1.00 & Ref. \\
\hline \multicolumn{7}{|c|}{ Eating difficulties due to oral health problems } \\
\hline Few or none & 1.00 & Ref. & 1.00 & Ref. & 1.00 & Ref. \\
\hline Some & 1.77 & $0.99,3.18$ & 1.42 & $0.77,2.64$ & $1 \cdot 16$ & $0.57,2.34$ \\
\hline Many & $2 \cdot 17^{\star}$ & $1.16,4.06$ & 1.64 & $0.90,2.99$ & 1.21 & $0.59,2.48$ \\
\hline \multicolumn{7}{|l|}{ Symptoms of depression } \\
\hline Absence & 1.00 & Ref. & 1.00 & Ref. & 1.00 & Ref. \\
\hline Presence & $4.05^{\star}$ & $2.48,6.62$ & $3.33^{*}$ & $2.03,5.48$ & $2 \cdot 56^{\star}$ & $1.55,4.23$ \\
\hline \multicolumn{7}{|l|}{ Functional status (IADL) } \\
\hline Independent & 1.00 & Ref. & 1.00 & Ref. & 1.00 & Ref. \\
\hline Dependent & $6 \cdot 92^{*}$ & $3.15,15 \cdot 20$ & $5 \cdot 63^{*}$ & $2.54,12.45$ & $4.70^{\star}$ & $1.98,11.11$ \\
\hline \multicolumn{7}{|l|}{ Loneliness feelings } \\
\hline Absence & 1.00 & Ref. & 1.00 & Ref. & 1.00 & Ref. \\
\hline Presence & $2 \cdot 14^{\star}$ & $1.25,3.66$ & $1.91^{\star}$ & $1 \cdot 12,3 \cdot 26$ & 1.33 & $0.74,2.37$ \\
\hline
\end{tabular}

IADL, instrumental activities of daily living.

${ }^{*} P<0.05$.

†OR were estimated using a logistic regression model using complex sample analysis (for model 3 , Nagelkerke pseudo $R^{2}=0.302$ ).

$\ddagger$ Model 1, crude OR; model 2, adjusted for sex, age and financial situation; model 3, adjusted for variables in model 2 plus appetite, eating difficulties due to oral health problems, symptoms of depression, functional status and loneliness feelings.

$\S$ Participants with missing values for any of the independent variables were excluded from the model $(n 55)$.

$\|$ Not adjusted for financial situation.

knowledge, the distribution of older adults living in nursing homes in Portugal by sex, marital status and educational level has not been previously described. If our results are compared with the most updated national census information from 2011, the proportions of women, individuals aged 85 years or over, widows/widowers and individuals who attended primary school or less are higher in our sample of institutionalised participants than in the total Portuguese population aged $\geq 65$ years $(72.8 v$. $58.1 \%, 49.2$ v. $11.7 \%, 64.6$ v. $30.6 \%$ and 89.0 v. $82.5 \%$, respectively) ${ }^{(48)}$. Regarding socio-economic characteristics, it is also worth mentioning that near $40 \%$ considered their financial situation as hard or very hard.

It is well known that malnutrition prevalence differs according to the setting, context, population studied, and instruments used to assess nutritional status. In a review including twenty-nine studies published between 2013 and 2014, the prevalence of malnutrition in nursing homes ranged between $1.5 \%(n$ 1614) and 66.5\% ( $n$ 5334), with an overall mean of $21 \cdot 4 \%{ }^{(23)}$. Furthermore, a systematic review including seventy-seven studies published between 1990 and 2011 reported that MNA $^{\circledR}$ was the most frequently used tool to assess nutritional status. Still, the MNA $^{\circledR}$ malnutrition prevalence rate was wide (0-71\%), although in most of the studies, 20 to $39 \%$ of residents were malnourished and 47 to $62 \%$ were at risk ${ }^{(49)}$. In thirty-two studies of institutionalised older persons using $\mathrm{MNA}^{\circledR}$ ( $n$ 6821), the prevalence of malnutrition was 21 $(\mathrm{SD} 0.5) \%$ (range $=5-71 \%)$ and the prevalence of risk of malnutrition was 51 (sD $0 \cdot 6$ ) \% (range $=27-70 \%$ ). The heterogeneity concerning level of dependence and health status of older residents contributes to explain the wide range observed in the prevalence of malnutrition risk/ malnutrition in this population ${ }^{(36)}$. 
Bedridden, demented and physically or mentally impaired individuals are expected to be in worse nutritional status ${ }^{(9)}$. These factors may partly explain the low percentages of malnourished individuals detected in the present study, because those considered severely impaired and unable to answer a long questionnaire were excluded. Results reported here are in line with findings from a similar study conducted in a representative sample of the Spanish nursing home population without cognitive impairment: $2 \cdot 8 \%$ were malnourished, $37.3 \%$ were at malnutrition risk, and the prevalence was lower for men and younger participants ${ }^{(50)}$. In contrast, a Belgium study in which $75.6 \%$ of the nursing home residents had a cognitive problem reported that one in five $(19.4 \%)$ residents was malnourished ${ }^{(51)}$. These results support that a low cognitive status is associated with malnutrition, which might arise by both loss of appetite or indifference to food. These individuals may also forget to eat or not be able to plan and prepare their meals or feed themselves ${ }^{(4,6)}$.

In the present study, $3.3 \%$ of the participants were classified as underweight according to WHO cut-offs, $40.5 \%$ presented a BMI between 25.0 and $29.9 \mathrm{~kg} / \mathrm{m}^{2}$, and $28.6 \%$ were classified as obese. The percentage of older adults at risk of malnutrition (according to $\left.\mathrm{MNA}^{(}\right)$) was higher among those having normal weight $(\mathrm{BMI}=18 \cdot 5-$ $24.9 \mathrm{~kg} / \mathrm{m}^{2}$ ) than among those with pre-obesity (BMI= $25 \cdot 0-29.9 \mathrm{~kg} / \mathrm{m}^{2}$; WHO criteria). The MNA ${ }^{\circledR}$ considers several factors that contribute to malnutrition other than BMI to assess the nutritional status. In fact, there has been much controversy in the scientific community with respect to the use of BMI and the most adequate cut-offs, especially for older adults. First, there are limitations to accurately measure weight and height among older persons, given functional limitations to correctly stand by her/ himself, as well as due to shrinkage and vertebral collapse. In these cases, the possible bias introduced by use of alternative measures might counteract the misclassification of malnutrition due to direct measurements errors ${ }^{(52)}$. Second, BMI does not capture changes in body weight or body composition. Weight loss, particularly progressive loss of lean body mass, is common in older ages and of utmost importance to the timely identification and reversal of malnutrition risk ${ }^{(53)}$. Furthermore, there is good evidence that BMI values below $21 \cdot 0-22 \cdot 0 \mathrm{~kg} / \mathrm{m}^{2}$ are associated with significant health status deterioration and increased mortality in older people ${ }^{(13,54)}$. Also, nutrient and fat storage of 'pre-obese' individuals, as considered by the WHO criteria, may protect them from reduction in functional strength, malnutrition and sarcopenia ${ }^{(13)}$. Moreover, micronutrient deficiencies and fat-free mass index were not evaluated in the present study, which could shed light on the coexistence of overweight and risk of malnutrition. Given the aforementioned, when considering risk of morbidity and mortality, it is probable that the BMI cut-offs for older adults may be different from those for younger adults ${ }^{(54)}$. While this is an open discussion, the WHO criteria as used in the present study have been considered standard, allowing comparisons between international studies and the compilation in pooled data analysis ${ }^{(55,56)}$.

Both physiological changes associated with ageing and pathology-related factors may cause oral health problems that possibly influence food choices and lead to reduced food intake ${ }^{(4)}$. Despite more than half of our sample being 85 years old or over, almost $70 \%$ reported few or no difficulties eating due to oral health problems. Regarding appetite, about a quarter $(24.2 \%)$ reported little or no appetite in the last $7 \mathrm{~d}$. Lack of appetite may be a mediating factor explaining the high risk of malnutrition among depressed persons, due to deterioration of social networks, as well as increased concentrations of serotonin and corticotropin-releasing factor ${ }^{(4)}$.

After adjusting for potential confounders, the logistic regression model showed that older adults reporting no or little appetite, symptoms of depression, as well as those who were more dependent in daily living activities were also at higher odds of being malnourished or at risk of malnutrition. These findings are supported by relevant literature ${ }^{(49,57)}$. In fact, depression, impaired function and poor oral intake have been consistently associated with weight loss, low BMI or poor nutrition $^{(10)}$. Interestingly, loneliness feelings were no longer significant when all studied potential confounders were included in the model. It might be hypothesised that other psychological variables are obscuring the effect of loneliness on malnutrition; however, for this to be ascertained, further investigation in the nursing home setting is required.

In a study conducted in Italian nursing homes, malnourished participants had more severe cognitive impairment, were more depressed and had lower scores in activities of daily living and physical performance (representing worse functional status) in comparison to their well-nourished counterparts ${ }^{(28)}$, similarly to the present study. However, Italian subjects had a lower prevalence of depression symptomatology as assessed by the GDS (women: $27 \cdot 3 \%$, men: $22 \cdot 2 \%$ ) than reported in our study. Among the disorders affecting mental health, depression has been considered the most common cause of involuntary weight loss and malnutrition in older adults ${ }^{(6)}$. Lower functional status to perform IADL such as shopping, preparing food and feeding (as measured by the Lawton scale) independently increases the risk of worse nutritional status, as found in the present study. Moreover, there is evidence that older adults who present cumulatively a risk of malnutrition and dependence in activities of daily living show the lowest survival rate ${ }^{(58)}$.

Inevitably, the present study has limitations that should be kept in mind when interpreting the results. First, except for anthropometric measurements, collected data 
represent self-reported answers, thus relying on memory or knowledge, participants' experiences and interpretations, and are eventually subject to some social desirability bias. To minimise this bias, participants were screened for cognitive function (assessed with MMSE) to understand if they could provide reliable answers to the survey, except for $\mathrm{MNA}^{\circledR}$ that could be completed using formal caregivers' help and/or professional judgement (all interviewers were nutritionists). Second, the exclusion criteria had an important contribution to the results regarding nutritional status, because those who were bedridden or who could not understand and/or answer the questionnaires were not enrolled in the study. This methodological decision took account of the sampling effort, the available resources for the estimated sample size and the tools that were chosen. The selected instruments were designed to be administered to the participant her/himself and not to a proxy, as they require very personal answers depending on feelings and emotions. This is especially relevant for depression symptoms and loneliness perception. Third, some anthropometric measurements were not performed in standardised conditions. For example, mainly due to mobility limitations and to ensure participants' comfort, some of them were not wearing light clothes only. Finally, as in all cross-sectional studies, there are some methodological limitations. For example, we cannot presume causal relationships between nutritional status and depression or IADL.

The current study has several strengths. First is the low refusal rate to participate in the study, both from institutions and residents. Second is its nationally representative nature and the large sample size with no upper age limit. Finally, the use of MNA ${ }^{\circledR}$ full form allowed the assessment of nutritional status, which was then explained by a model including a specific set of sociodemographic, socioeconomic and psychosocial variables. This combination of factors has rarely been used to further understand malnutrition in nursing homes.

Our findings reassert that malnutrition, especially risk of malnutrition, is prevalent among nursing home residents in Portugal. This finding is particularly relevant because it was observed among participants who were not bedridden nor with dementia. On the one hand, this may imply an underestimation of malnutrition prevalence; on the other, it singles out a group of individuals with potential for improvement. To better understand the magnitude of this problem, future studies should include those bedridden or with dementia. Increased awareness and objective quantifications of nutritional status may prompt more proactive policies at national and local levels, which will guide effective interventions aimed at reducing malnutrition risk factors such as depression and lower functional status. Such policies should consider all material and human resources required to routinely conduct effective evaluations and interventions in nursing homes.

\section{Acknowledgements}

Acknowledgements: The authors acknowledge the Instituto de Segurança Social for providing the list of all Portuguese nursing homes, and staff of Faculdade de Medicina, Universidade de Lisboa for the administrative support and financial management. The authors acknowledge the IAN-AF team for its support with the development of data collection software and critical discussion of the study protocol. The authors also acknowledge the interviewers who collected the data among nursing home residents, the managers and administrations of the participating nursing homes, and all participants. The authors acknowledge Joana Costa for reviewing this manuscript. Financial support: The present project (136SI5) was granted by the Public Health Initiatives Programme (PT06), financed by EEA Grants Financial Mechanism 2009-2014. T.M. is supported by a PhD Scholarship (grant number SFRH/BD/ 117884/2016) from Fundação para a Ciência e a Tecnologia (FCT) through national funds (MCTES). Funding organisations had no role in the design/conduct of the study, the collection/analysis/interpretation of the data, or the preparation/review/approval of the manuscript. Conflict of interest: None. Authorship: T.M., C.P.-P., O.S., V.A., B.G., N.M., P.J.N. and J.G.C. contributed to the initial study design protocol and to obtain funding. T.M., C.P.-P., N.S.-S., O.S., V.A., B.G., N.M., P.J.N., A.Y., A.By., A.Be., T.F.A. and J.G.C. contributed to the final research protocol. T.M. coordinated the study implementation and wrote the first draft of this manuscript. O.S. was involved in all methodological details of the project. N.S.-S. contributed to the development of data collection software. C.L. participated in the design of tools for data collection. All authors contributed to the further discussion of the manuscript. All authors read and approved the final manuscript. Ethics of human subject participation: This study was conducted according to the guidelines laid down in the Declaration of Helsinki and all procedures involving human subjects were approved by the National Data Protection Committee and the Faculdade de Medicina da Universidade de Lisboa Ethics Committee. Nursing homes' managers provided formal informed consent and authorisation to participate in the project. Written informed consent was obtained from all subjects. When detecting malnutrition, participants received an informative form about their status, being advised by interviewers (nutritionists) to contact their medical doctors.

\section{Supplementary material}

To view supplementary material for this article, please visit https://doi.org/10.1017/S1368980018002318 


\section{References}

1. World Health Organization (2003) Diet, Nutrition and the Prevention of Chronic Diseases. Joint WHO/FAO Expert Consultation. WHO Technical Report Series no. 916. Geneva: WHO

2. World Health Organization (2015) World Report on Ageing and Health. Geneva: WHO.

3. Amarantos E, Martinez A \& Dwyer J (2001) Nutrition and quality of life in older adults. J Gerontol A Biol Sci Med Sci 56, 54-64.

4. Wysokiński A, Sobów T, Kłoszewska I et al. (2015) Mechanisms of the anorexia of aging - a review. Age (Dordr) 37, 9821

5. Saarela RKT, Lindroos E, Soini H et al. (2016) Dentition, nutritional status and adequacy of dietary intake among older residents in assisted living facilities. Gerodontology 33, 225-232.

6. John BK, Bullock M, Brenner L et al. (2013) Nutrition in the elderly. Frequently asked questions. Am J Gastroenterol 108, 1252-1266.

7. Guerchet M, Prina M, Prince M et al. (2014) Nutrition and Dementia: A Review of Available Research. London: Alzheimer's Disease International.

8. Jyrkkä J, Mursu J, Enlund H et al. (2012) Polypharmacy and nutritional status in elderly people. Curr Opin Clin Nutr Metab Care 15, 1-6.

9. Meijers JMM, Schols JMGA \& Halfens RJG (2014) Malnutrition in care home residents with dementia. $J$ Nutr Health Aging 18, 595-600.

10. Tamura BK, Bell CL, Masaki KH et al. (2013) Factors associated with weight loss, low BMI, and malnutrition among nursing home patients: a systematic review of the literature. J Am Med Dir Assoc 14, 649-655.

11. Tani Y, Kondo N, Takagi D et al. (2015) Combined effects of eating alone and living alone on unhealthy dietary behaviors, obesity and underweight in older Japanese adults: results of the JAGES. Appetite 95, 1-8.

12. Donini LM, Scardella P, Piombo L et al. (2013) Malnutrition in elderly: social and economic determinants. J Nutr Health Aging 17, 9-15.

13. Soenen S \& Chapman IM (2013) Body weight, anorexia, and undernutrition in older people. J Am Med Dir Assoc 14, 642-648.

14. European Society for Clinical Nutrition and Metabolism (2011) Diagnosis of malnutrition - screening and assessment. In Basics in Clinical Nutrition, 4th ed., pp. 21-32 [L Sobotka, SP Allison, A Forbes et al., editors]. Prague: Galén.

15. Cederholm T, Bosaeus I, Barazzoni R et al. (2015) Diagnostic criteria for malnutrition - an ESPEN consensus statement. Clin Nutr 34, 335-340.

16. Neyens J, Halfens R, Spreeuwenberg M et al. (2013) Malnutrition is associated with an increased risk of falls and impaired activity in elderly patients in Dutch residential long-term care (LTC): a cross-sectional study. Arch Gerontol Geriatr 56, 265-269.

17. Lesourd B (2004) Nutrition: a major factor influencing immunity in the elderly. J Nutr Health Aging 8, 28-37.

18. Rasheed S \& Woods RT (2013) Malnutrition and quality of life in older people: a systematic review and meta-analysis. Ageing Res Rev 12, 561-566.

19. Norman K, Pichard C, Lochs $\mathrm{H}$ et al. (2008) Prognostic impact of disease-related malnutrition. Clin Nutr 27, 5-15.

20. Alexopoulos GS (2005) Depression in the elderly. Lancet 365, 1961-1970.

21. Kassebaum NJ, Arora M, Barber RM et al. (2016) Global, regional, and national disability-adjusted life-years (DALYs) for 315 diseases and injuries and healthy life expectancy (HALE), 1990-2015: a systematic analysis for the Global Burden of Disease Study 2015. Lancet 388, 1603-1658.
22. Inzitari M, Doets E, Bartali B et al. (2011) Nutrition in the age-related disablement process. J Nutr Health Aging 15, 599-604.

23. Bell CL, Lee ASW \& Tamura BK (2015) Malnutrition in the nursing home. Curr Opin Clin Nutr Metab Care 18, 17-23.

24. Cereda E (2012) Mini nutritional assessment. Curr Opin Clin Nutr Metab Care 15, 29-41.

25. Morley JE, Caplan G, Cesari M et al. (2014) International survey of nursing home research priorities. J Am Med Dir Assoc 15, 309-312.

26. Gabinete de Estratégia e Planeamento (2015) Carta social Rede de equipamentos e serviços 2015. Lisbon: Ministério do Trabalho, Solidariedade e Segurança Social (MTSSS).

27. Gabinete de Estratégia e Planeamento (2009) A dependência: o apoio informal, a rede de serviços e equipamentos $e$ os cuidados continuados integrados. Lisbon: Ministério do Trabalho, Solidariedade e Segurança Social (MTSSS).

28. Donini LM, Poggiogalle E, Molfino A et al. (2016) MiniNutritional Assessment, Malnutrition Universal Screening Tool, and Nutrition Risk Screening Tool for the nutritional evaluation of older nursing home residents. J Am Med Dir Assoc 17, 959.e11-e18.

29. Madeira T, Peixoto-Plácido C, Goulão B et al. (2016) National survey of the Portuguese elderly nutritional status: study protocol. BMC Geriatr 16, 139.

30. World Medical Association (2013) World Medical Association Declaration of Helsinki: ethical principles for medical research involving human subjects. JAMA 310, 2191-2194.

31. Lopes C, Torres D, Oliveira A et al. (2018) National Food, Nutrition, and Physical Activity Survey of the Portuguese general population (2015-2016): protocol for design and development. JMIR Res Protoc 7, e42.

32. Folstein MF, Folstein SE \& McHugh PR (1975) 'Mini-mental state'. A practical method for grading the cognitive state of patients for the clinician. J Psychiatr Res 12, 189-198.

33. Guerreiro M, Silva AP, Botelho MA et al. (1994) Adaptação à população portuguesa da tradução do 'Mini Mental State Examination' (MMSE). Rev Port Neurol issue 1, 9-10.

34. Rubenstein LZ, Harker JO, Salvà A et al. (2001) Screening for undernutrition in geriatric practice: developing the shortform mini-nutritional assessment (MNA-SF). J Gerontol $A$ Biol Sci Med Sci 56, M366-M372.

35. Vellas B, Villars H, Abellan G et al. (2006) Overview of the MNA - its history and challenges. J Nutr Health Aging 10, 456-463.

36. Guigoz Y (2006) The Mini Nutritional Assessment (MNA) review of the literature - what does it tell us? J Nutr Health Aging 10, 466-485.

37. Stewart AD, Marfell-Jones M, Olds T et al. (2011) International Standards for Anthropometric Assessment. Lower Hutt, New Zealand: International Society for the Advancement of Kinanthropometry.

38. Guerra RS, Fonseca I, Pichel F et al. (2014) Hand length as an alternative measurement of height. Eur J Clin Nutr 68, 229-233.

39. Kaiser MJ, Bauer JM, Ramsch C et al. (2009) Validation of the Mini Nutritional Assessment short-form (MNA-SF): a practical tool for identification of nutritional status. J Nutr Health Aging 13, 782-788.

40. World Health Organization (1995) Physical Status: The Use and Interpretation of Anthropometry. Report of a WHO Expert Committee. WHO Technical Report Series no. 854. Geneva: WHO.

41. Sheikh JI \& Yesavage JA (1986) Geriatric Depression Scale: recent evidence and development of a shorter version. Clin Gerontol 5, 165-173.

42. Pocklington C, Gilbody S, Manea L et al. (2016) The diagnostic accuracy of brief versions of the Geriatric Depression Scale: a systematic review and meta-analysis. Int J Geriatr Psychiatry 31, 837-857. 
43. Lawton MP \& Brody EM (1969) Assessment of older people: self-maintaining and instrumental activities of daily living. Gerontologist 9, 179-186.

44. Russell DW (1996) UCLA Loneliness Scale (Version 3): reliability, validity, and factor structure. J Pers Assess 66, 20-40.

45. Pocinho M, Farate C \& Amaral Dias C (2000) Validação psicométrica da escala UCLA - loneliness para idosos Portugueses. Interações 18, 65-77.

46. Strathmann S, Lesser S, Bai-Habelski J et al. (2013) Institutional factors associated with the nutritional status of residents from 10 German nursing homes (ErnSTES study). $J$ Nutr Health Aging 17, 271-276.

47. Suominen M, Muurinen S, Routasalo P et al. (2005) Malnutrition and associated factors among aged residents in all nursing homes in Helsinki. Eur J Clin Nutr 59, 578-583.

48. Instituto Nacional de Estatística (2012) CENSOS 2011. http://censos.ine.pt/xportal/xmain?xpid=CENSOS\&xpgid= censos_quadros (accessed August 2017).

49. Bell CL, Tamura BK, Masaki KH et al. (2013) Prevalence and measures of nutritional compromise among nursing home patients: weight loss, low body mass index, malnutrition, and feeding dependency, a systematic review of the literature. J Am Med Dir Assoc 14, 94-100.

50. Serrano-Urrea R \& Garcia-Meseguer MJ (2013) Malnutrition in an elderly population without cognitive impairment living in nursing homes in Spain: study of prevalence using the Mini Nutritional Assessment test. Gerontology 59, 490-498.

51. Verbrugghe M, Beeckman D, Van Hecke A et al. (2013) Malnutrition and associated factors in nursing home residents: a cross-sectional, multi-centre study. Clin Nutr 32, 438-443.

52. Gavriilidou NN, Pihlsgård M \& Elmståhl S (2015) High degree of BMI misclassification of malnutrition among Swedish elderly population: age-adjusted height estimation using knee height and demispan. Eur J Clin Nutr 69, 565571.

53. Chang S-H, Beason TS, Hunleth JM et al. (2012) A systematic review of body fat distribution and mortality in older people. Maturitas 72, 175-191.

54. Peter RS, Mayer B, Concin H et al. (2015) The effect of age on the shape of the BMI-mortality relation and BMI associated with minimum all-cause mortality in a large Austrian cohort. Int J Obes (Lond) 39, 530-534.

55. Veronese N, Cereda E, Solmi M et al. (2015) Inverse relationship between body mass index and mortality in older nursing home residents: a meta-analysis of 19,538 elderly subjects. Obes Rev 16, 1001-1015.

56. Flegal KM, Kit BK, Orpana H et al. (2013) Association of allcause mortality with overweight and obesity using standard body mass index categories. JAMA 309, 71-82.

57. Favaro-Moreira NC, Krausch-Hofmann S, Matthys C et al. (2016) Risk factors for malnutrition in older adults: a systematic review of the literature based on longitudinal data. Adv Nutr 7, 507-522.

58. Naseer M \& Fagerström C (2015) Prevalence and association of undernutrition with quality of life among Swedish people aged 60 years and above: results of the SNAC-B study. J Nutr Health Aging 19, 970-979. 